\title{
Publisher Correction: Harnessing the intracellular triacylglycerols for titer improvement of polyketides in Streptomyces
}

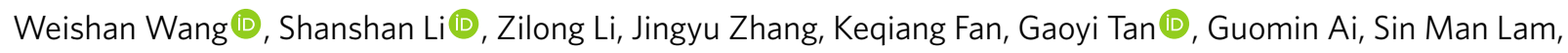
Guanghou Shui, Zhiheng Yang, Hongzhong Lu, Pinjiao Jin, Yihong Li, Xiangyin Chen, Xuekui Xia, Xueting Liu, H. Kathleen Dannelly, Chen Yang, Yi Yang (D), Siliang Zhang, Gil Alterovitz, Wensheng Xiang (i) and Lixin Zhang (1)

Correction to: Nature Biotechnology https://doi.org/10.1038/s41587-019-0335-4, published online 9 December 2019.

In the version of this article initially published online, the bottom half of the orange arrow connecting FAs to $\beta$-oxidation via acyl-CoA synthetase was missing in Fig. 4a. The error has been corrected in the print, PDF and HTML versions of the article.

Published online: 18 December 2019

https://doi.org/10.1038/s41587-019-0389-3

๑ The Author(s), under exclusive licence to Springer Nature America, Inc. 2019 